\title{
Clear Expansion Plate: A Novel Technique
}

\author{
Ahmad Behroozian ${ }^{1, \odot}$ \\ ${ }^{1}$ Department of Orthodontics, Dental School, Tabriz University of \\ Medical Sciences, Tabriz, Iran
}

Eur J Gen Dent 2021;10:120-122.

\begin{abstract}
Address for correspondence Ahmad Behroozian, DDS, MSc, Department of Orthodontics, Faculty of Dentistry, Tabriz University of Medical Sciences, Tabriz, 51639-16737, Iran (e-mail: ahmadbehroozian@yahoo.com).
\end{abstract}

\begin{abstract}
Keywords

- clear plate

- crossbite

- expansion

- mixed dentition

- primary dentition

Objectives Posterior crossbite is one of the most common orthodontic problems in primary and mixed dentition that induces deleterious and permanent effects on dentition, dentoskeletal structures, and esthetics. Early correction of unilateral and bilateral posterior crossbite is necessary to prevent these problems. Early intervention with removable Schwarz appliance is one of the useful appliances to correct posterior crossbite, but it has its own disadvantages.

Materials and Methods In this article, we have presented a new appliance and method of fabrication to aid the expansion of the maxilla.

Results The presented "clear expansion plate" that is made by thermoplastic sheets maintains all of the positive properties of the Schwarz plate and has several additional advantages.

Conclusion After being tested in clinical trials, traditional expansion plate can be replaced by clear expansion plate.
\end{abstract}

\section{Introduction}

According to the literature, posterior crossbite is one of the most common types of malocclusion in deciduous and mixed dentitions. Its prevalence ranges from 8 to $16 \%,{ }^{1,2}$ and more than $90 \%$ of posterior crossbites expressing a functional shift into a unilateral crossbite. ${ }^{3}$

A crossbite is seldom self-correcting and usually worsens with growth, and can give rise to a lot problems like asymmetric condylar positioning, facial asymmetry, altered archforms, dentoalveolar compensations in all three planes of space, neuromuscular disharmony, and obstructing maxillary development. Therefore, early intervention in the primary dentition is necessary. ${ }^{2,4}$

Treatment for posterior crossbites requires maxillary expansion to achieve appropriate arch width and allow normal closure patterns of the mandible without functional deviation. The effects of early treatment in primary dentition appear to be stable in the majority of patients. It has been reported that the effects of crossbite treatment in the primary dentition is stable and the permanent teeth erupted in a correct position in $84 \%$ without relapse. ${ }^{5}$

It has been shown that the young age of the patients is important to achieve good outcomes, because when treatment is performed in very young children midpalatal suture can be stimulated before increasing interdigitation. ${ }^{6,7}$ In preschool age removable appliances can induce dental and skeletal changes. ${ }^{6}$ And are the device of choice in these age groups. ${ }^{8}$ The retention of the removable appliances is low because of the specific anatomic form of the crown of the primary teeth.

Commonly used split-acrylic-type appliances (e.g., Schwarz plate) has some disadvantages like poor patient cooperation in wearing the appliance because of the appearance of the wires, lack of appliance retention, and fitting. ${ }^{9}$

To overcome the limitations of traditional removable appliances in expansion of the maxillary arch in primary and early mixed dentition, we have described a new removable appliance and method of fabrication with the name of
DOI https://doi.org/ 10.1055/s-0041-1733303 ISSN 2320-4753
(C) 2021. European Journal of General Dentistry.

This is an open access article published by Thieme under the terms of the Creative Commons Attribution-NonDerivative-NonCommercial-License, permitting copying and reproduction so long as the original work is given appropriate credit. Contents may not be used for commercial purposes, or adapted, remixed, transformed or built upon. (https://creativecommons.org/licenses/by-nc-nd/4.0/).

Thieme Medical and Scientific Publishers Pvt. Ltd. A-12, 2nd Floor, Sector 2, Noida-201301 UP, India 
the "clear expansion plate" made by thermoplastic sheets. It maintains all of the positive properties of Schwarz plate as well as several additional advantages.

\section{Method of Fabrication}

Form a thick thermoformable sheet using standard vacuum-forming procedures, on the maxillary stone models, similar to clear retainer fabrication ( - Fig. 1). Remove a window in the palatal region of the retainer, then fix a midpalatal screw in the window with self-cured acrylic (-Fig. 2). Cut the retainer from the midline with a disc (Video 1) and

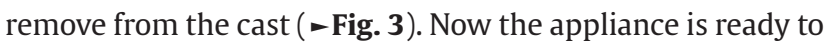
be used ( - Fig. 4 ). The preliminary intraoral photos show the success of the appliance in expansion of the maxilla ( - Fig. 5 ).

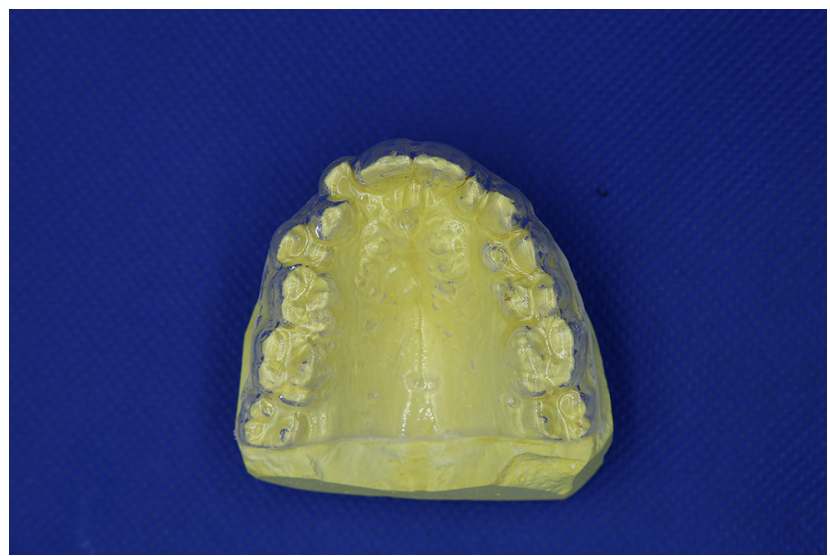

Fig. 1 The retainer is made by thick thermoformable sheet.

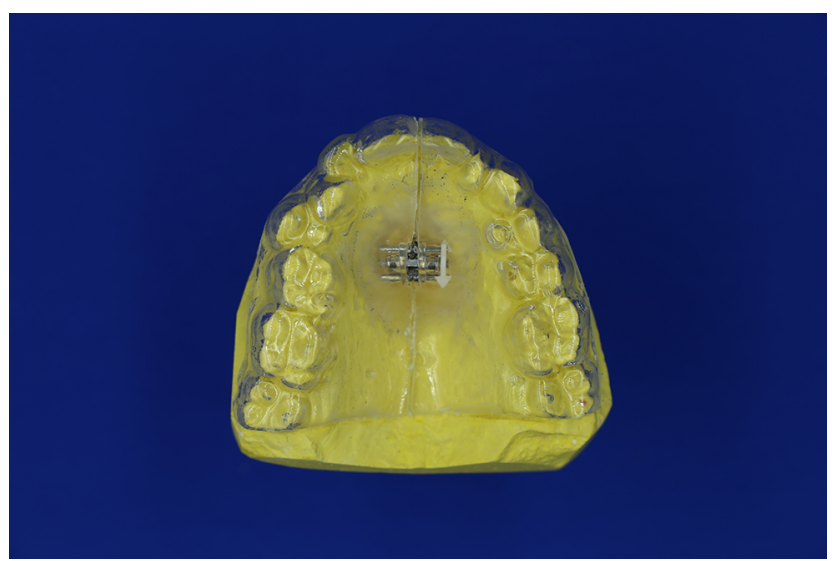

Fig. 2 Expansion screw is fixed by self-cure acrylic.

\section{Video 1}

How to fabricate clear expansion plate. Online content including video sequences viewable at: https:// www.thieme-connect.com/products/ejournals/ html/10.1055-s-0041-1733303.

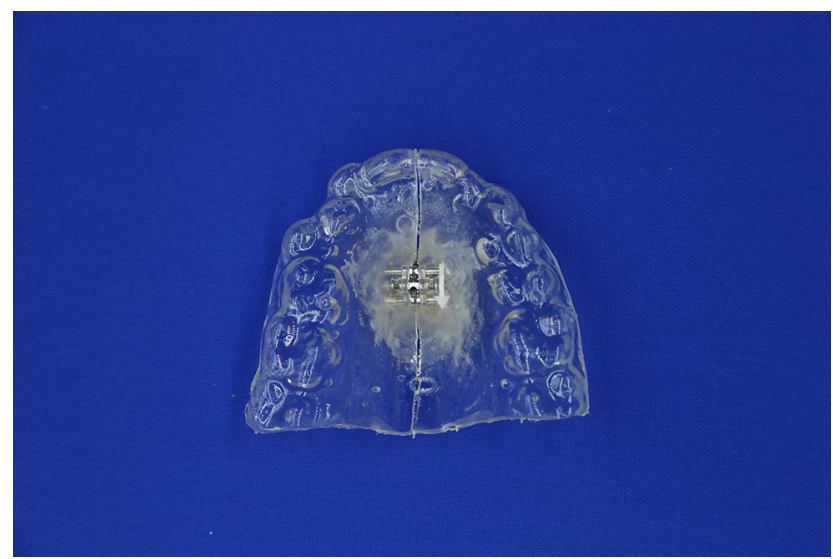

Fig. 3 Midpalatal cut is made.

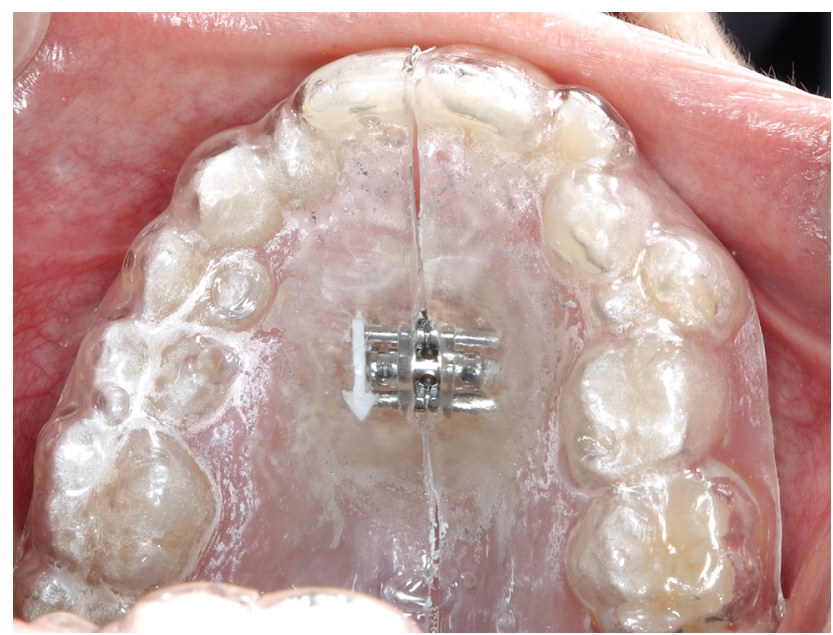

Fig. 4 The clear expansion plate is delivered to the patient.

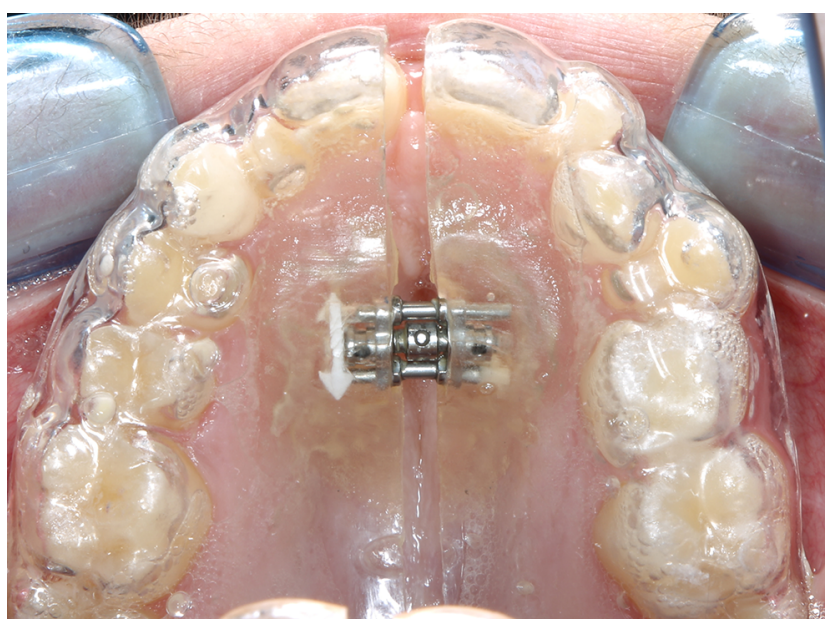

Fig. 5 The effect of the appliance after few weeks.

\section{Discussion}

We have introduced a new technique by incorporating a midpalatal expansion screw in clear retainer to improve upon patient cooperation, reduce fabrication time, and improve chair-side efficiency. 
Indeed, the research explored changing the original design of the traditional split-acrylic-type appliances (e.g., Schwarz plate) by replacing the acrylic plate and wire clasps with clear retainers.

Schwarz plate had somewhat lower success rates at 50 to $70 \%{ }^{2}$ Less than $100 \%$ success rate can be attributed to poor patient cooperation and lack of retention. Also, wire elements of traditional removable plates namely "arrowheads of Adams' clasp" and "the loop of the labial bow" can irritate the soft tissue of the patient and cause painful ulcers that inhibit the child to wear the appliance. ${ }^{10}$

The presented "clear expansion plate" has no wire elements on the surface of the teeth; therefore, its appearance is better than the traditional acrylic plate. Therefore, higher patient acceptance and higher wear time is anticipated. Also, wearing the present appliance is more comfortable to tolerate because it has no wires to damage the soft tissue of the patients, which in turn, increases wear time.

Because of its anatomic form, the crown of the primary teeth cannot provide much retention to the traditional removable appliances. But in clear expansion plate all surfaces of the teeth is involved and higher degree of retention is anticipated; in addition, involvement of all teeth guarantees the retention because extraction or exfoliation of any teeth cannot jeopardize the retention.

From cost-effectiveness point of view, clear expansion plate is less time consuming in office because there is no need to adjust the wire clasps each session. In addition, this technique saves the time for laboratory technician because time-consuming wire bending process is eliminated. The time spent for "wire bending plus acrylic plate pouring and polishing" in traditional method is not comparable with fabricating a "clear retainer" in the present technique.

One of the drawbacks of the traditional removable appliances which are used in expansion of the upper arch is that they can exert only tipping force. Full coverage of the clinical crowns can control the tipping of the anterior teeth, although further investigation is necessary to prove this allegation.

In summary, the present technique provides several advantages over commonly used traditional split-acrylic-type appliance, including:

- The absence of visible components in the appliance makes it esthetic and for the patient to wear full time.

- It has no clasps so reduced tissue irritation makes it more comfortable for the patient.

- Reduced chair-time, as there are no wire clasps to be adjusted.

- Reduced laboratory time, as the wire bending process has been eliminated.

- Full crown engagement of the teeth is anticipated to make more bodily movement of the teeth.

- Since all of the teeth are involved in retention, it is anticipated to be more retentive and the exfoliation of any primary teeth should not jeopardize the retention.

\section{Suggestion for Further Assessment}

We presented only a novel opinion so we propose to design a multicenter randomized clinical trial to compare the clear expansion plate to the traditional appliance.

\section{Author's Contribution}

A.B. conceived the initial research idea, prepared the manuscript, and reviewed the final manuscript prior to submission.

\section{Financial Support}

None.

\section{Conflict of Interest}

None declared.

\section{Acknowledgments}

I would like to acknowledge Milad Hemmatiyan who prepared the 3 dimensional virtual models and the video file. Thanks also to Amir Faturechi, the technician of dental laboratory, who warmly helped us in making the true models.

\section{References}

1 Dimberg L, Lennartsson B, Söderfeldt B, Bondemark L. Malocclusions in children at 3 and 7 years of age: a longitudinal study. Eur J Orthod 2013;35(1):131-137

2 Bell RA, Kiebach TJ. Posterior crossbites in children: developmental-based diagnosis and implications to normative growth patterns. Semin Orthod 2014;20:77-113

3 Egermark-Eriksson I. Malocclusion and some functional recordings of the masticatory system in Swedish schoolchildren. Swed Dent J 1982;6(1):9-20

4 Tollaro I, Defraia E, Marinelli A, Alarashi M. Tooth abrasion in unilateral posterior crossbite in the deciduous dentition. Angle Orthod 2002;72(5):426-430

5 Schröder U, Schröder I. Early treatment of unilateral posterior crossbite in children with bilaterally contracted maxillae. Eur J Orthod 1984;6(1):65-69

6 Defraia E, Marinelli A, Baroni G, Tollaro I. Dentoskeletal effects of a removable appliance for expansion of the maxillary arch: a postero-anterior cephalometric study. Eur J Orthod 2008;30(1):57-60

7 Jimenez-Valdivia LM, Malpartida-Carrillo V, RodríguezCárdenas YA, Dias-Da Silveira HL, Arriola-Guillén LE. Midpalatal suture maturation stage assessment in adolescents and young adults using cone-beam computed tomography. Prog Orthod 2019;20(1):38

8 Jacobs SG. Teeth in cross-bite: the role of removable appliances. Aust Dent J 1989;34(1):20-28

9 Goulart CS, Thiesen G, Nuernberg NJ. GCS expansion appliance: fixed-removable expander. Dental Press J Orthod 2011;16:79-86

10 Baricevic M, Mravak-Stipetic M, Majstorovic M, Baranovic M, Baricevic D, Loncar B. Oral mucosal lesions during orthodontic treatment. Int J Paediatr Dent 2011;21(2):96-102 